\title{
Diseño y validación de un nuevo registro clínico de enfermería, para la continuidad de los cuidados y seguridad del paciente en hemodiálisis
}

\author{
Miguel Ángel Cuevas-Budhart' ${ }^{1}$, Blanca Ruth González Martínez², Elsa Alvarez Bolaños ${ }^{3}$, María Juana Barrera \\ García ${ }^{4}$, Alina de Almeida Souza ${ }^{5}$, Máximo A. González Jurado ${ }^{6}$, Mercedes Gómez del Pulgar García Madrid ${ }^{5}$ \\ ${ }^{1}$ Hospital General Regional n 1 "Vicente Guerrero". Acapulco, Guerrero. México. Instituto Español de Investiga- \\ ción Enfermera.Madrid. España \\ ${ }^{2}$ Unidad de Medicina Familiar n 29 con Unidad Médica de Atención Ambulatoria. IMSS. Guerrero. México \\ ${ }^{3}$ Coordinación Delegacional de Enfermería en Atención Médica. IMSS. Guerrero. México \\ ${ }^{4}$ Facultad de Enfermería No. 2 UAGro. Posgrado de Gestión y Docencia. México \\ ${ }^{5}$ Instituto Español de Investigación enfermera del Consejo General de Enfermería. Madrid. España \\ ${ }^{6}$ Consejo General de Enfermería. Madrid. España
}

Como citar este artículo:

Cuevas-Budhart MA, González-Martínez BR, Álvarez-Bolaños E, Barrera-García MJ, de Almeida-

Souza A, González-Jurado MA, Gómez del Pulgar-García-Madrid M. Diseño y validación de un nuevo registro clínico de enfermería, para la continuidad de los cuidados y seguridad del paciente en hemodiálisis. Enferm Nefrol. 2019 Abr-Jun;22(2):168-75

\section{Resumen}

Introducción: Diseñar y Validar una herramienta para la mejora del proceso de atención en enfermería en una unidad de hemodiálisis de un hospital de segundo nivel.

Material y Método: Se desarrolló un estudio de mejora de la calidad de la atención, en el Hospital General Regional $n^{\circ} 1$ "Vicente Guerrero" en Guerrero, (México), entre mayo-agosto de 2017. Se modificó la hoja de registros clínicos de enfermería de la unidad de hemodiálisis en dos etapas. Para la etapa de diseño se realizó un grupo focal con expertos y para la validación de contenido se aplicó el método Delphi. Los datos recolectados se procesaron y analizaron en el programa estadístico SPSS V.22.

Resultados: El 70\% de los expertos consideró el instrumento como bueno en cuanto a la calidad del regis-

Correspondencia:

Miguel Ángel Cuevas-Budhart

Paseo de la Esperanza 8, 4B esc. D portal 4.28005 Madrid

E-mail: angel_budhart@hotmail.com tro. Hubo una discrepancia en el diseño encontrándose como parcialmente eficiente ( $70 \%$ ) ya que cada experto evaluó de acuerdo al área que dominaba. Se incorporaron todas las observaciones realizadas en el diseño final.

Conclusión: El instrumento se considera válido, proporcionando información adecuada del paciente previo al tratamiento hemodialítico, lo cual satisface los requerimientos legales respecto a la continuidad de los cuidados.

PALABRAS CLAVE: atención al paciente; calidad; seguridad del paciente; continuidad; hemodiálisis; registro de enfermería.

Design and validation of a new nursing clinical registry for the continuity of care and patient safety in hemodialysis

\section{Abstract}

Objective: To design and validate a tool for improving nursing care process in a hemodialysis unit of a secondary level hospital. 
Material and Method: A quality improvement study was developed at Regional General Hospital $\mathrm{N}^{\circ} 1$ Vicente Guerrero in Guerrero, Mexico, between mayaugust 2017. Hemodialysis nurse clinical record sheet was modified in two stages. For the design stage, a focus group with experts was carried out and for content validation, Delphi method was applied. The collected data were processed and analyzed with statistics program SPSS V.22.

Results: $70 \%$ of experts considered the instrument as good in terms of quality of the record. There was a discrepancy in the design, considered partially efficient (70\%), since each expert evaluated according the area that dominated. All the observations made were included in the final design.

Conclusion: The instrument is valid, giving the right information about the patient prior to hemodialysis, satisfying legal requirements regarding the continuity of care.

KEYWORDS: patient care; quality; patient safety; continuity; hemodialysis; nursing record.

\section{Introducción}

Actualmente, la seguridad del paciente forma parte indisoluble de la calidad en la atención sanitaria. De acuerdo con la definición de seguridad del Instituto de Medicina de Estados Unidos lo define como: "conjunto de elementos y metodologías basadas en evidencia científicamente probada, para minimizar el riesgo de sufrir un evento adverso en el proceso de atención de salud o mitigar sus consecuencias ${ }^{\prime \prime}$.

Dicho lo anterior, los profesionales de salud y en particular el profesional de enfermería, juega un papel importante en la continuidad de los cuidados y la seguridad del paciente, esto implica, entre otras cosas, desarrollar estrategias de mejora y continuidad del cuidado con la finalidad de garantizar y optimizar los servicios prestados ${ }^{2}$.

En el desempeño de su profesión, Enfermería aporta procesos de mantenimiento y recuperación de la salud del individuo, familia y comunidad en diferentes etapas de la vida. La formación académica evoluciona a la par del avance tecnológico, con el objetivo de ofrecer servicios de salud de calidad y seguridad acorde a las necesidades y problemas de salud que afecta a la población ${ }^{3}$.
La comunicación como parte del quehacer enfermero es considerado una pieza clave para la continuidad de los cuidados. Los registros clínicos de enfermería son instrumentos o herramientas orientadas a documentar de forma permanente los cuidados en un marco ético legal cuya información señale las acciones de enfermería en relación con el cuidado integral del sujeto. Esta debe ser escrita, detallada, completa, concisa, y apropiada sobre el cuidado del paciente lo que significa que las observaciones deben ser actuales e inmediatas a los cuidados para evitar la omisión o duplicación de la información y a su vez transmitir un orden lógico ${ }^{4,5}$. Su diseño y cumplimiento dependen del modelo de cuidados, distribución de trabajo y asignación de pacientes, así, como, de otras circunstancias como puede ser el profesional y el tipo de pacientes ${ }^{6}$.

Sin embargo, la calidad de los registros clínicos de enfermería se puede cuestionar por diversas razones: 1) la obsolescencia en el formato y forma de recolección de información; 2) cambio continuo del conocimiento y las acciones de cuidados basados en evidencia $y, 3$ ) el desconocimiento de la importancia legal de contar con un lenguaje apropiado para el cumplimiento del registro de Enfermeria ${ }^{1}$. La atención a la persona de cuidado en el servicio de hemodiálisis no es ajena a cualquiera de estos tres escenarios, debido a la compleja combinación de procedimientos tecnológicos e interacciones humanas, existiendo el riesgo de producirse Eventos Adversos (EA); no obstante, existen EA que son prevenibles con una correcta valoración, por ello, en este contexto, deben ser considerados como una condición que requiere un ambiente humanístico, como un abordaje integral encaminado al dialogo y el trabajo colaborativo entre profesionales que permita innovar y actualizar los procesos del servicio para garantizar la seguridad del paciente y mejorar la calidad ${ }^{7}$.

En base a lo anterior, se ha evidenciado la importancia de mejorar el formato de los registros clínicos de enfermería, tomando como base el proceso de atención de enfermería. Utilizando el modelo de valoración de enfermería los 11 patrones funcionales de salud de Marjory Gordon la cual permite obtener una importante cantidad de datos relevantes del paciente de manera ordenada y organizada y a su vez facilita el análisis, identificando factores de riesgo que comprometan la seguridad del paciente, y previniendo EA que se presentan en la unidad de Hemodiálisis ${ }^{8}$.

De acuerdo a la N0M-003-SSA3-2010 "Para la práctica de Hemodiálisis ${ }^{\prime \prime}$ no se cumple con esta evaluación. Sin 
embargo, el profesional de enfermería de la unidad de hemodiálisis del IMSS en su responsabilidad con el cuidado, tiene un compromiso con el paciente y la sociedad, que requiere y demanda competencias específicas, identificar riesgos y adminístralos oportunamente con el objetivo de prevenir errores, principalmente antes de causar daño.

Por todo ello, se planteó como principal objetivo diseñar y validar una herramienta para la mejora del proceso de atención en enfermería en una unidad de hemodiálisis de un hospital de segundo nivel.

\section{Material y Método}

Para el diseño y validación de un nuevo registro clínico de enfermería se realizó un estudio de mejora a la calidad con un abordaje mixto, con una fase cualitativa utilizando para ello un enfoque fenomenológico, mediante un grupo focal, como técnica de recogida de información, cuyo facilitador fue el investigador principal10,11; y una fase cuantitativa, para la validación de contenido por jueces expertos mediante método Delphi en una segunda etapa ${ }^{12,13}$.

En la primera fase, participaron en el estudio 10 enfermeras asistenciales y 1 jefe de servicio de la unidad de hemodiálisis en el periodo de mayo-noviembre. Para la segunda fase, la muestra se tomó a toda la población que cumpliera con los criterios de selección, los cuales fueron: personal de enfermería ambos sexos, con grado de licenciatura, experiencia en la unidad de hemodiálisis, valoración y cuidados del paciente renal, por último, que aceptaran precipitar en el estudio.

También participaron como asesores durante todo el estudio, 6 expertos y 2 investigadores en las distintas fases de desarrollo; identificación, diseño, y evaluación de la herramienta debido a su formación aspectos como valoración aplicada en proceso de atención de enfermería, nefrología, EA y diseño de herramientas de mejora de la calidad.

Para el diseño del registro se siguió el procedimiento de acuerdo a referentes de diseño y validación de formularios de enfermería el cual se visualiza en la Figura $\mathbf{l}$.

- Fase 1: Correspondió a la fase cualitativa la cual comprendió el análisis de la literatura en diversas bases de datos como PubMed, MEDLINE, Biblioteca Cochrane, CONRyCIT entre otras, así como la recogida de información de experiencias clínicas de los enfermeros asistenciales en la unidad de Hemodiálisis, mediante grupo focal. Tras la reunión en la cual se les explico los objetivos del estudio y

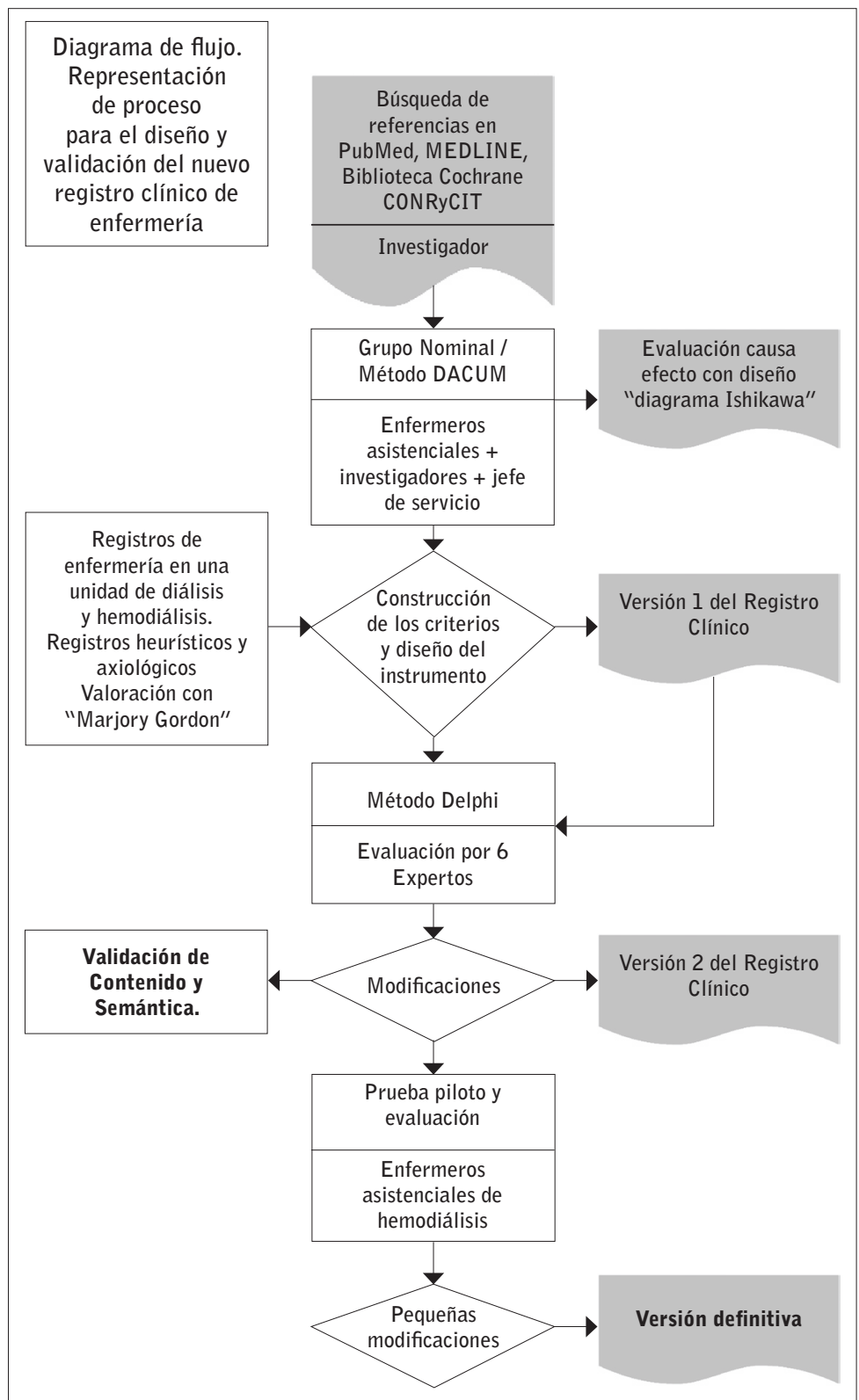

Figura 1. Proceso de diseño y validación de un nuevo registro clinico de enfermería. Fuente: realizado por el autor.

se les solicito apoyo y colaboración en el mismo, se identificaron áreas de oportunidad que pudieran coadyuvar a la mejora de la calidad en la atención mediante la evaluación causa efecto con el "diagrama Ishikawa" en el que se establecieron como principales problemas: Baja calidad de los registros clínicos para la continuidad de la atención del paciente, falta de registros del proceso de atención de enfermería para la prevención de complicaciones y deficiente registro de eventos adversos.

De acuerdo a los resultados de la primera reunión los investigadores diseñaron una primera versión de un nue- 
vo registro clínico de enfermería, haciendo énfasis en los problemas detectados. En la segunda reunión se propuso un instrumento diseñado por los investigadores, el cual incluía la implementación del modelo de valoración de los patrones funcionales de salud de Marjory Gordon ${ }^{14,15}$, registro de EA, eventos centinela y cuasifalla ${ }^{16,17}$. Todos los datos se analizaron y retroalimentaron de acuerdo a las necesidades y solicitudes de los enfermeros asistenciales de la unidad de Hemodiálisis quedando así una primera versión del registro clínico de Enfermería.

- Fase 2: Correspondió a la etapa cuantitativa; Para verificar la validez de contenido de la primera versión, fue sometida validación de 6 expertos, quienes realizaron un análisis de manera global mediante análisis semántico y de aplicabilidad, mediante una encuesta que constaba de evaluación de la calidad, estructura, contenido y utilidad del registro clínico de enfermería, obteniéndose así la versión número 2.

- Fase 3: esta fase correspondió a la capacitación, prueba piloto y validación del registro clínico por enfermeras nefrológicas de la unidad de hemodiálisis. Para lo cual, se realizó una reunión en cada turno de la unidad de hemodiálisis para realizar la capacitación al personal sobre la cumplimentación del registro, proceso de atención de enfermería, importancia del registro clínico y $E A$, y resolución de las dudas.

El pilotaje se realizó durante 6 sesiones en pacientes de hemodiálisis. La evaluación global se efectuó de acuerdo a la versión 2 de la herramienta la cual se repartió a las enfermeras nefrológicas de la unidad. Posteriormente a la prueba piloto, estas respondieron a un cuestionario con una escala tipo Likert con unja puntuación de 1 a 4 , sobre la estructura y calidad del proceso de atención de enfermería del registro, donde 1 correspondía a la peor valoración y 4 a la más completa. El segundo instrumento se calificó del 1 a 5, donde 1 era estar totalmente en desacuerdo y 5 totalmente de acuerdo, sobre la pertinencia, sencillez y aplicabilidad de la herramienta de mejora y la comprensibilidad, claridad y adecuación de sus elementos o criterios de valoración. Además, las enfermeras asistenciales que participaron anexaron comentarios y sugerencias para la modificación y mejora del registro clínico de enfermería. Posterior al pilotaje, se realizaron pequeñas modificaciones, quedando así la versión definitiva.

Los datos recolectados se procesaron y analizaron en el programa estadístico SPSS V.22. Las variables cualitativas se expresan como una distribución de frecuencias y las variables cuantitativas como la media \pm la desviación estándar de la media.

Consideraciones éticas: El estudio fue presentado y aprobado por el comité de Ética de la Delegación Guerrero del Instituto Mexicano del Seguro Social (IMSS), que, tras su revisión del proyecto dictamino como favorable al no implicar riesgo en los participantes. Otorgando el número de registro R-2017-2001-23.

\section{Resultados}

La validación del nuevo registro clínico de enfermería fue realizada por expertos de manera global y se ajustaron los cambios pertinentes de acuerdo a la retroalimentación semántica y de aplicabilidad de la herramienta.

En la etapa cualitativa, en relación a la fase del proceso enfermero, se destacó: "cumple con el proceso enfermero adecuado para la valoración, atención y evaluación de las intervenciones, sin embargo, es importante considerar el tiempo de cumplimentación para la atención individualizada del paciente en hemodiálisis" (experto 3).

En cuanto a la funcionalidad en Hemodiálisis, se destacó: "creo conveniente agregar los valores que trae de línea blanca y la línea roja, glóbulos blancos, hematocrito, hemoglobina, plaquetas, para ello poder gestionar si hay necesidad de transfundir o infundir algún hemoderivado" (experto 5).

Para la fase cuantitativa, en la cual se validó la herramienta, se contó con 10 enfermeros (8 enfermeras y 2 enfermeros), con un promedio de edad de 36 años, el $50 \%$ como tenía como máximo grado de estudios superiores, la Licenciatura; tal como se refleja en la Tabla 1.

Respecto al grado de aceptación del proceso de mejora, enfocado a la valoración de Margory Gordon, se encontró que el $90 \%$ del personal de hemodiálisis cuenta con los cursos sobre dicho tema (ver Tabla 2).

Durante el tiempo que se mantuvo en implementación la hoja de registros clínicos de enfermería, se identificaron los patrones funcionales de mayor afectación, siendo el patrón de Eliminación e intercambio el de mayor alteración (23\%). Los datos se muestran en la Tabla 3.

Los EA registrados durante la prueba piloto fueron; Hipertensión $(41,9 \%)$, Hipotensión $(25,6 \%)$, Disnea $(9,3 \%)$, y en menos medida Dolor torácico, Cefalea, Ta- 
Tabla 1. Datos laborales del personal asistencial que participo en el estudio.

\begin{tabular}{llll}
\hline Variable & No. & $\%$ & X+DE \\
\hline Edad & & & \\
$\quad 23$ a 50 años & & & $36 \pm 10,27$ \\
Género & & & \\
$\quad$ Masculino & 2 & 20 & \\
$\quad$ Femenino & 8 & 80 & \\
Grado académico & & & \\
$\quad$ Licenciado en Enfermería & 5 & 50 & \\
$\quad$ Pos técnico en Nefrología & 3 & 30 & \\
$\quad$ Posgrado en nefrología & 1 & 10 & \\
$\quad$ Maestría & 1 & 10 & \\
Años de antigüedad & & & $10 \pm 6,1$ \\
$\quad 3$ a 20 años & & & \\
$\quad$ Turno & & & \\
$\quad$ Matutino & 5 & 50 & \\
$\quad$ Vespertino & 5 & 50 & \\
Servicio de procedencia & & & \\
$\quad$ Urgencias & 1 & 10 & \\
$\quad$ Medicina Interna & 2 & 20 \\
$\quad$ Pediatría & 2 & 20 \\
Diálisis peritoneal & 5 & 50 \\
\hline
\end{tabular}

Tabla 2. Experiencia sobre el proceso de atención de enfermería.

\begin{tabular}{lll}
\hline Variable & No. & $\%$ \\
\hline $\begin{array}{l}\text { Curso sobre PAE } \\
\text { Si }\end{array}$ & 9 & 90 \\
No & 1 & 10 \\
\hline Cantidad de Cursos & & \\
$\quad 1$ a 3 & 5 & 50 \\
4 a 6 & 3 & 30 \\
$>7$ & 2 & 20 \\
Tipo de curso & & \\
$\quad$ Facultad de Enfermería & 3 & 30 \\
$\quad$ Curso monográfico IMSS & 5 & 50 \\
Capacitación en el Servicio & 2 & 20 \\
Duración del curso & & \\
$\quad 1$ a 8 horas & 3 & 30 \\
9 a 20 horas & 4 & 40 \\
$\quad>21$ horas & 3 & 30 \\
\hline Conocimiento sobre el modelo de valoración & & \\
Patrones funcionales de Marjory Gordon & & \\
Si & 9 & 90 \\
No & 1 & 10 \\
\hline
\end{tabular}

quicardia y Dolor abdominal, con $2,3 \%$ cada uno. También se registraron 2 caídas en la prueba piloto.

De acuerdo a la evaluación del registro de enfermería en la dimensión de calidad de la herramienta. Se observa una evaluación como buena calidad del $70 \%$ en opinión de los
Tabla 3. Patrones funcionales alterados durante la valoración del paciente en hemodiálisis.

\begin{tabular}{lcc}
\hline \multicolumn{1}{|l}{ Variable } & No. & $\%$ \\
Patrones funcionales alterados & & \\
\hline Patrón 3 Eliminación e intercambio. & 89 & 23 \\
\hline Patrón 2 Nutricional Metabólico. & 83 & 22 \\
\hline Patrón 4 Actividad/Ejercicio. & 70 & 18 \\
\hline Patrón 1 Percepción de la Salud. & 68 & 18 \\
\hline Patrón 5 Sueño/Reposo. & 31 & 8 \\
\hline Patrón 7 Autopercepción/Autoconcepto. & 12 & 3 \\
\hline Patrón 6 Cognitivo Perceptual. & 11 & 3 \\
\hline Patrón 8 Rol Relaciones. & 8 & 2 \\
\hline Patrón 10 Tolerancia al Estrés. & 6 & 2 \\
\hline Patrón 11 Valores y Creencias. & 3 & 1 \\
\hline
\end{tabular}

expertos. Respecto al diseño del nuevo registro de enfermería hubo una discrepancia, encontrándose como un diseño parcialmente eficiente de contenido $(70 \%)$ seguido de Diseño eficiente de contenido (20\%), esto debido a que cada experto dio su punto de vista de acuerdo al área que domina, sin embargo, se atendió cada observación, quedando una versión final o versión definitiva (Figura 2).

\section{Discusión}

La mejora de la calidad depende en gran medida de un trabajo en equipo ya que genera expectativas positivas para el personal que presta servicios de salud. Ante la propuesta de mejora, la participación fue activa en las fases de identificación del problema principal, en la unidad de hemodiálisis. Los cambios necesarios para lograr una mejora al problema identificado, implica directamente al servicio de enfermería. En lo que respecta a la evaluación del diseño hubo discrepancias, ya que cada experto dio su punto de vista de acuerdo al área que dominaba; realizándose cambios de acuerdo a la evaluación de cada uno de ellos, para obtener así la versión definitiva del documento.

El instrumento diseñado y validado, intenta responder a la problemática identificada en el servicio de Hemodiálisis, cumpliendo con los criterios del enfoque enfermero, en sus etapas de valoración, diagnostico, intervención y evaluación, mediante la valoración de los patrones funcionales de Marjory Gordon, así como también intenta coadyuvar al registro de EA, cumpliendo 


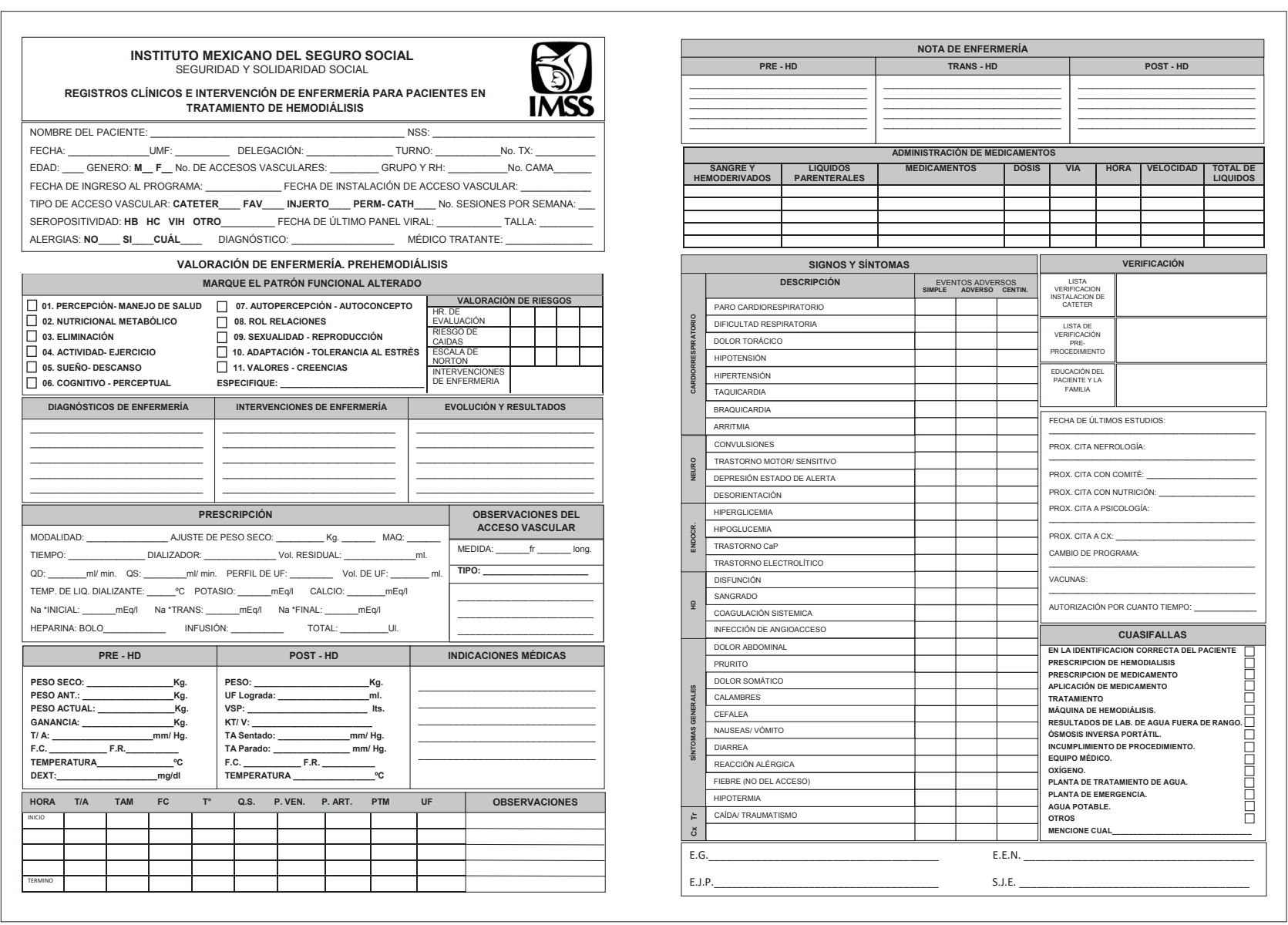

Figura 1. Registro clínico de enfermería modificado. Fuente: realizado por el autor.

con lo estipulado en el documento "Acciones Esenciales para la Seguridad del Paciente" de la Organización Mundial de la Salud ${ }^{18,19}$.

Al utilizar una metodología cualitativa y cuantitativa, proporciona un método exhaustivo, eficiente y psicométricamente sólido para medir la salud desde el punto de vista del profesional de Enfermeria y de los expertos en diferentes procesos. Por lo cual, consideramos nuestro instrumento como válido, ya que es la base para la planificación de la atención, proporcionando información adecuada del paciente previo a su tratamiento hemodialítico, lo cual responde a los requerimientos legales respecto a la continuidad de los cuidados, estipulado por la NOM004-SSA3-2012 , ya que proporciona información para una correcta gestión clínica, evaluación y seguridad del pacientel, bajo las normas de bioseguridad son las medidas y disposiciones que buscan proteger la vida a través del logro de actitudes y conocimientos adecuados, del profesional de Enfermería, lo cual, es imprescindible para la mejora de la calidad general en la atención a los pacientes.
Algunos autores advierten, que para que exista un registro clínico de enfermería exitoso se necesita contar con la participación del personal de enfermería asistencial ${ }^{20,21}$, sin embargo, el contar con profesionales expertos en diversas áreas como gerencia, calidad, seguridad del paciente, herramientas de calidad, proceso de atención de enfermería y el asistencial, proporciona un mejor acoplamiento al registro clínico de enfermería, ya que al haber sido diseñado de acuerdo a la estrategia de este estudio, permite que el instrumento sea identificado por el personal asistencial como exitoso ya que manifiesta las necesidades expresadas por el personal que trabaja en unidad de hemodiálisis.

Hay que resaltar que los profesionales de enfermería expertos en el área de nefrología respondieron positivamente a la fase de identificación de puntos clave para la elaboración del nuevo registro clínico, tras la primera elaboración de la prueba Delphi, así como en la prueba piloto, participaron con un carácter proactivo en la fase del proceso de mejora y continuidad del cuidado del paciente que 
se somete a hemodiálisis. Pensamos que esta participación, puede mejorar su desempeño, siendo de importancia para la calidad asistencial de la unidad de hemodiálisis.

Sin embargo, la mejora de la continuidad de los cuidados y la calidad de los registros clínicos no solo depende del personal que trabaja en la unidad, sino también del apoyo de los directivos para la toma de decisiones adecuadas para garantizar el bienestar y satisfacción del paciente. Cabe señalar las mejoras que puedan causar conflicto al inicio, pueden resultar una estrategia de valioso interés para el trabajo en equipo y mejora en la calidad asistencial.

\section{Limitaciones del estudio}

La principal limitación del estudio, se encuentra relacionado con la falta de tiempo para a evaluar la implementación, ya que convergen en el tiempo de evaluación por parte del comité científico y de investigación, para la autorización de campo clínico.

A la vista de los resultados de este estudio, podemos concluir que el instrumento se considera válido, proporcionando información adecuada del paciente previo al tratamiento con hemodiálisis, lo cual satisface los requerimientos legales respecto a la continuidad de los cuidados. Además, se recomienda, elaborar la fase de implementación, para evaluar los resultados de dicha herramienta; así como elaborar ciclos de mejora con metodología en otros servicios, ya que permite vislumbrar el desarrollo y evaluación de dicha estrategia, entendiéndose la importancia de evaluar actividades rutinarias, especialmente aquellas invasivas que exigen un elevado conocimiento y grado de responsabilidad del personal que trabaja en dichos servicios.

\section{Conflictos de interés}

Los autores declaran no tener ningún conflicto de intereses en lo que corresponde a la investigación, la autoría o la publicación de este artículo.

\section{Agradecimientos}

El presente trabajo fue realizado gracias al apoyo del equipo de Enfermeras de la unidad de Hemodiálisis del Hospital General Regional No. 1 "Vicente Guerrero" por su apoyo, disponibilidad y compromiso, así, como al grupo de expertos, por su tiempo, dedicación y retroalimentación en este proceso de mejora en la calidad de la aten- ción. También, se expresa un agradecimiento al Consejo Nacional de Ciencia y Tecnología (CONACyT) México, y al Instituto Español de Investigación Enfermera (IE) del Consejo General de Enfermería de España (CGE), por su preocupación y apoyo para que la Enfermería se mantenga siempre a la vanguardia, caminando con paso firme hacia la calidad.

\section{Recibido: 04-10-18 \\ Revisado: 05-12-18 \\ Modificado: 25-01-19 \\ Aceptado: 15-02-19}

\section{Bibliografía}

1. Torres Santiago LM, Zárate Grajales MRA, Matus Miranda MR. Calidad de los registros clínicos de enfermería: Elaboración de un instrumento para su evaluación. Enfermería Univ [Internet]. 2011;8(1):17-25. [Consultado 04 septiembre 2018]. Disponible en: http://www.revistas.unam. $\mathrm{mx} /$ index.php/reu/article/view/25467.

2. Grajales RZ. La gestión del cuidado de enfermeria. Scielo. 2004;13:44-5.

3. Programa Nacional de Salud 2007-2012 Por un México sano: construyendo alianzas para una mejor salud [Internet]. Mexico City, Mexico: Author. Retrieved August. 2007. 185. p. [Consultado 04 septiembre 2018]. Disponible en: https://bit. ly/2ZYMRvk.

4. Sánchez DH, Calero MÁR, Navarro MJG, Herrezuelo GB. Elaboración de un registro enfermero para hemodiálisis según la norma IS0 9001:2005. Rev la Soc Esp Enferm Nefrol [Internet]. 2007;10(1):1522. [Consultado 22 junio 2018]. Disponible en: https://bit.ly/2vHuX2b.

5. Suárez-Villa M. Registros de Enfermería como Evidencia del Cuidado. Ciencia e Innovación en Salud [Internet]. 2013;1(2). [Consultado 9 octubre 2018]. Disponible en: https://bit.ly/2IR9oB0.

6. Hernández-Corral S, Hernández-Mayén L, Pérez-Benavidez $L$, Retiz-Ortiz AD, Uribe-Benavides 
B, Vázquez-Santos L. Repercusión de un Programa de Mejora Continua en los Registros de Enfermería. Enf Neurol Enfermería Neurológica Enf Neurol [Internet]. 2010;9(1):13-6. [Consultado 04 septiembre 2018]. Disponible en: https://bit.ly/2LqJIkL.

7. Román CAL. La seguridad del paciente, una responsabilidad en el acto del cuidado. Vol. 22, Revista Cubana de Enfermeria. 2006.

8. Fajardo-Dolci G, Rodríguez-Suárez J, Campos-Castolo $M$, Carrillo-Jaimes A, Zavala-Suárez E, Aguirre-Gas $\mathrm{H}$. Lineaminetos generales para el cuidado de la seguridad del paciente. Rev CONAMED [Internet]. 2008;13(Julio-Septiembre):38-46. [Consultado 10 septiembre 2018]. Disponible en: http:// www.conamed.gob.mx/publicaciones/pdf/REVISTA_JUL-SEP_2008.pdf

9. Fallis A. Norma Oficial Mexicana NOM -003-SSA32010, Para I apráctica de la hemodiálisis. J Chem Inf Model. 2013;53(9):1689-99.

10. McLafferty I. Focus group interviews as a data collecting strategy. Journal of Advanced Nrsing [Internet]. 2004;48(2):187-94. [Consultado 22 junio 2018]. Disponible en: https://bit.ly/2E4UJCn.

11. Webb C, Kevern J. Focus groups as a research method: A critique of some aspects of their use in nursing research. J Adv Nurs. 2001;33(6):798-805.

12. Pill J. The Delphi method: Substance, context, a critique and an annotated bibliography. Socioecon Plann Sci. 1971;5(1):57-71.

13. Astigarraga E. El método delphi. Techniques [Internet]. 2006;1-14. [Consultado 09 octubre 2018]. Disponible en: http://www.gtic.ssr.upm.es/encuestas/delphi.htm.

14. Femeba F, Luis J, Suarez Á. Manual de valoración de patrones funcionales. Man Valoración Patrones Funcionales. 2010;5:1-26.
15. Andrés-Galache B. Diagnósticos de enfermería en pacientes con insuficiencia renal crónica en hemodiálisis. Rev Soc Española Enfermería Nefrológica [Internet]. 2004;7(3):158-63. [Consultado 4 septiembre 2018]. Disponible en: http://scielo.isciii.es/ $\mathrm{pdf} / \mathrm{nefro} / \mathrm{v} 7 \mathrm{n} 3 / \mathrm{art02}$.pdf

16. Hauck K, Zhao X, Jackson T. Adverse event rates as measures of hospital performance. Health Policy (New York). 2012;104(2):146-54.

17. Tennankore KK, D'Gama C, Faratro R, Fung S, Wong $\mathrm{E}$, Chan CT. Adverse technical events in home hemodialysis. Am J Kidney Dis. 2014;65(1):116-21.

18. Rojas-Armadillo ML, Jiménez-Báez MV, Chávez-Hernández MM, González-Fondón A. Validación de un sistema de registro de eventos adversos en atención primaria Validation of an adverse event reporting system in primary care. Aportaciones Orig Rev Med Inst Mex Seguro Soc. 2016;54(3):327-33.

19. Anglès $R$, Llinás $M$, Alerany $C$, García MV. Sistema de notificación genérico y gestión de incidentes: Implantación y acciones de mejora derivadas para la seguridad del paciente. Med Clin (Barc). 2013;140(7):320-4.

20. Urquhart C, Currell R, Grant MJ, Hardiker NR. Nursing record systems: effects on nursing practice and healthcare outcomes. Cochrane Database Syst Rev [Internet]. 2009; (1):CD002099. [Consultado 09 octubre 2018]. Disponible en: https://bit.ly/2YbKovH.

21. Aparecida J, Cerullo B, da Silva Bastos Cerullo JA, de Almeida Lopes Monteiro da Cruz D. Clinical reasoning and critical thinking. Rev Lat Am Enfermagem [Internet]. 2010;18(1):124-9. [Consultado 09 octubre 2018]. Disponible en: http://www.scielo.br/ pdf/rlae/v18n1/19.pdf

Este artículo se distribuye bajo una Licencia Creative Commons Atribución-NoComercial 4.0 Internacional. https://creativecommons.org/licenses/by-nc/4.0/

Open Access (c) (7) (8) 